\title{
Acute presentation of deep vein thrombosis in Achilles tendon rupture: A case report
}

\author{
Rafik Yassa, Kiran Singh Kandola, A Sharma
}

\begin{abstract}
Introduction: Rupture of the Achilles tendon is relatively common. High incidence of thromboembolism during the first eight weeks after Achilles tendon rupture has been reported in literature. The use of venous thromboembolism prophylaxis after an Achilles tendon rupture is controversial. Delayed deep vein thrombosis (DVT) is a well-known complication after operative and non-operative treatment of Achilles tendon rupture but it has not been reported as an acute presentation following achillies tendon rupture. Case Report: We report a case of DVT presenting acutely after Achilles tendon rupture. It presented with soft clinical signs. High index of suspicion was required to diagnose DVT. The authors could not find similar association between the two clinical problems reported in literature. Conclusion: We recommend that risk assessment should be undertaken in any patients following lower limb injury even if it is not a major injury and adequate thromboprophylaxis should be started
\end{abstract}

Rafik Yassa ${ }^{1}$, Kiran Singh Kandola ${ }^{1}$, A Sharma ${ }^{1}$

Affiliations: ${ }^{1}$ Registrar in Trauma and Orthopaedics, Trauma and Orthopaedic Department, Queen Elizabeth The Queen Mother hospital, St Peter's road, Margate, UK. CT9 4AN.

Corresponding Author: Rafik Yassa, Registrar in Trauma and Orthopaedics, Trauma and Orthopaedic department, Queen Elizabeth The Queen Mother hospital, St Peter's road, Margate, UK. CT9 4AN; Ph: 07956340789; Email: rafikragai@yahoo.com

Received: 21 May 2012

Accepted: 20 July 2012

Published: 01 February 2013 according to the risk rating when deciding on immobilization of the lower limb.

Keywords: Achilles tendon, Rupture, Venous thrombosis

$* * * * * * * * *$

Yassa R, Singh Kandola K, Sharma A. Acute presentation of deep vein thrombosis in achilles tendon rupture: A case report. International Journal of Case Reports and Images 2013;4(2):99-102.

$* * * * * * * * *$

doi:10.5348/ijcri-2013-02-270-CR-4

\section{INTRODUCTION}

Venous thromboembolism is a recognized complication after lower limb injury [1]. Rupture of the Achilles tendon is relatively common and deep vein thrombosis (DVT) is a well-known complication after operative or non-operative treatment of Achilles tendon rupture.

High incidence of thromboembolism during the first eight weeks after Achilles tendon rupture has been reported [2]. To the best of the authors' knowledge acute presentation of DVT after Achillis tendon rupture has not been reported in literature. The use of venous thromboembolism prophylaxis after an Achilles rupture is controversial. The rates of reported deep vein thrombosis (DVT) range from $6.3-34 \%$. There is no agreement regarding prophylactic therapy after an Achilles tendon rupture [3].

We present a case of acute presentation of Achilles tendon rupture with concomitant DVT. Such a diagnosis should be considered when assessing for a ruptured achilles tendon and planning the method of treatment. 


\section{CASE REPORT}

A 44-year-old female suffered forced dorsiflexion of the ankle, when she lost her footing on a step. Medical history revealed discectomy three years back. She was on regular use of paracetamol and non-steroidal antiinflammatory medication (ibuprofen) for residual low back pain. She was a smoker of 1 pack/day. On clinical examination in the emergency department, Achilles tendon was found to be tender on palpation. She was unable to stand on tip-toes. Simmonds-Thompson test was positive. The test involves having the patient lie prone on an examination bed or kneeling on a chair with both feet extending past the end of examining table or chair, on squeezing the calf muscles on the affected side by the examiner's hand, the foot will demonstrate plantar flexion if the tendon is intact. On the other hand, if tendon is ruptured, the foot will not demonstrate plantar flexion. This test may lose its accuracy one week after injury. A below knee back slab was applied with foot in equinus as a initial temporary measure.

Three days later, the patient was seen in the orthopedic clinic to decide on the definitive treatment, when it was noticed that the swelling had became diffuse involving the whole leg from the knee downwards which was more than that expected for Achilles tendon rupture alone. She also had slight calf tenderness. As this raised the additional possibility of deep venous thrombosis (DVT), a venous duplex scan as well as an ultrasound scan of the tendon was performed in the same day. The ultrasound scan revealed Achilles tendon rupture $5 \mathrm{~cm}$ above its insertion into the calcaneus with a gap of $1 \mathrm{~cm}$. The venous duplex scan also surprisingly revealed DVT of the popliteal and posterior tibial veins (Figure 1).

Because of the concomitant ipsilateral DVT, the decision was made to treat her non-operatively with a dorsal plaster of paris slab, first in equinus for three weeks, then followed by a further three weeks with the ankle in a neutral position. On hemotological advice, low molecular weight heparin in the therapeutic dose (1.5 mg/kg body weight) was administered for six weeks, followed by warfarin for six weeks at therapeutic levels (INR 2-3). The patient had a follow up in the orthopedic clinic till she had a complete recovery and healing of the Achilles tendon rupture but she continued her regular visits to the hematology clinic for about six months thereafter. There was no reported evidence of pulmonary embolism or any complication from either the DVT or its treatment.

\section{DISCUSSION}

Deep vein thrombosis is a recognized complication after an injury to the lower limb [1]. A prospective study reported high incidence of thromboembolism during the first eight weeks after an acute achilles tendon rupture [2], but no study has reported acute presentation of DVT after an achillis tendon rupture. Clinical diagnosis

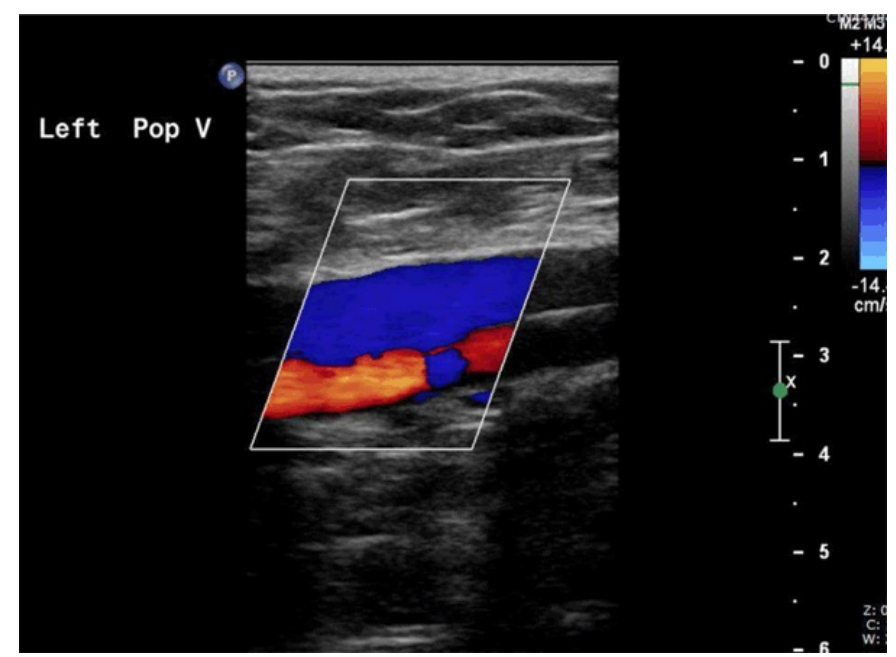

Figure 1: Duplex Scan Showing DVT of the popliteal and posterior tibial veins.

in the acute setting is difficult and unreliable due to pain, ecchymosis, tenderness and swelling from the trauma itself. In addition, ultrasonographic diagnosis in the acute setting involves some technical problems due to pain, hematoma and soft tissue oedema.

The DVT, pulmonary emboli (PE) and cardiovascular events are serious complications after orthopedic surgery. The risk of thromboembolism after acute achillies tendon rupture has been demonstrated in several studies [4-6]. Venous thromboembolism was demonstrated in $34 \%$ patients, two months after an acute total Achilles tendon rupture [2]. In a large retrospective study by Patel et al., the overall rates for DVT and PE after Achilles tendon ruptures were $0.43 \%$ and $0.34 \%$, respectively. Age older than 40 years, congestive heart failure, history of DVT or PE, obesity, and whether a patient had surgery; did not predict occurrence of DVT or PE (Table 1) [3].

However, after injury or surgery to the lower limbs the clinical diagnosis of DVT is fairly challenging. This is because of the combined factors of pain, tenderness, swelling and immobilization. Objective methods are required for DVT diagnosis. Moreover, the majority of DVTs are asymptomatic and the true incidence is unknown. A proportion of the clots in subtle DVTs can be complicated by PE [2].

The use of colored duplex sonography (CDS) to detect DVT, especially after trauma and surgery has been challenged $[7,8]$. In a recent study CDS was performed eight weeks after the injury to avoid the technical problems and ambiguity [2].

Thromboprophylaxis for lower limb injury was evaluated in several previous studies. In patients immobilized with plaster casts due to lower leg injuries, Kujath et al. demonstrated a reduction in the incidence of DVT when prophylactic treatment was used [9]. On the other hand, Patel et al. believe that routine use of anticoagulation might be unwarranted (Table 2) [3].

In United Kingdom, NICE provide clear guidelines for the thromboprophylaxis after minor surgery, lower limb injury and lower limb plaster casts (Figure 2) [10]. 
Table 1: Venous thromboembolism (VTE) risk assessment

\section{Patients who are at risk of VTE}

\section{Medical patients}

- If mobility significantly reduced for $\geq 3$ days, or

- If expected to have ongoing reduced mobility relative to normal state + any VTE risk factor

\section{VTE risk factors}

- Active cancer or cancer treatment

- Age $>60$ years

- Critical care admission

- Dehydration

- Known thrombophilias

- Obesity $\left(\mathrm{BMI}>30 \mathrm{~kg} / \mathrm{m}^{2}\right)$

- One or more significant medical comorbidities (for example, heart disease, metabolic, endocrine or respiratory pathologies, acute infectious diseases, inflammatory conditions etc.)

- Personal history or first-degree relative with a history of VTE

- Use of hormone replacement therapy

- Use of oestrogen-containing contraceptive therapy

- Varicose veins with phlebitis

\section{Surgical patients and patients with trauma}

- If total anaesthetic + surgical time $>90$ minutes, or

- If surgery involves pelvis or lower limb and total anaesthetic + surgical time $>60$ minutes, or

- If acute surgical admission with inflammatory or intraabdominal condition, or

- If expected to have significant reduction in mobility, or

- If any VTE risk factor present
Table 2: Risk of bleeding assessment

All patients who have any of the following are at risk of bleeding:

- Active bleeding

- Acquired bleeding disorders (such as acute liver failure)

- Concurrent use of anticoagulants known to increase the risk of bleeding (such as warfarin with INR >2)

- Lumbar puncture/epidural/spinal anaesthesia within the previous four hours or expected within the next 12 hours

- Acute stroke

- Thrombocytopenia (platelets $<0.75 \times 10^{5} / \mathrm{mm}^{3}$ )

- Uncontrolled systolic hypertension $(\geq 230 / 120$ $\mathrm{mmHg}$ )

- Untreated inherited bleeding disorders (such as hemophilia or von Willebrand's disease)

Balance the risk of VTE and bleeding before offering VTE prophylaxis

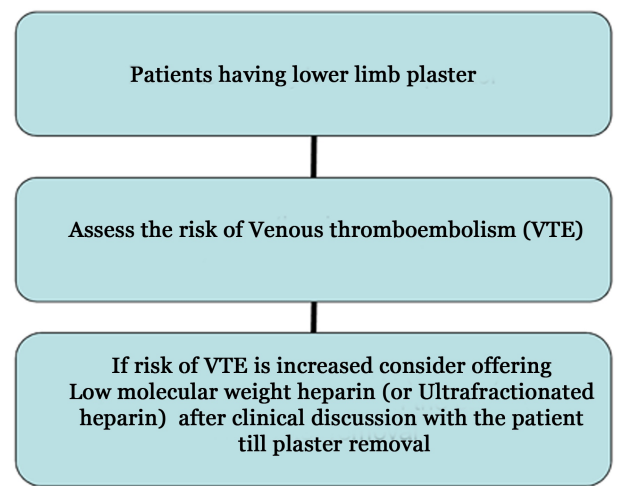

Figure 2: NICE algorithm for DVT prophylaxis in lower limb plaster. Venous thromboembolism, Low molecular weight heparin, Ultrfractionated heparin for patients with renal failure.

\section{CONCLUSION}

Deep vein thrombosis may presents acutely with lower limb injury. Risk assessment should be undertaken in any patient following lower limb injury even if it is not a major injury. Adequate thromboprophylaxis should be started, if it is indicated, when deciding on immobilization of the lower limb, either above or below knee immobilization, especially in plaster.

$$
* * * * * * * * *
$$

\section{Author Contributions}

Rafik Yassa - Conception and design, Acquisition of data, Drafting the article, revising it critically for important intellectual content and final approval of the version to be published

Kiran Singh Kandola - Conception and design, Acquisition of data, Drafting the article, revising it critically for important intellectual content and final approval of the version to be published

A Sharma - Conception and design, Acquisition of data, Drafting the article, revising it critically for important intellectual content and final approval of the version to be published

\section{Guarantor}

The corresponding author is the guarantor of submission.

\section{Conflict of Interest}

Authors declare no conflict of interest.

\section{Copyright}

(C) Rafik Yassa et al. 2013; This article is distributed under the terms of Creative Commons Attribution 3.0 
License which permits unrestricted use, distribution and reproduction in any means provided the original authors and original publisher are properly credited. (Please see www.ijcasereportsandimages.com/copyright-policy.php for more information.)

\section{REFERENCES}

1. Yusof MI. Acute Pulmonary embolism following Achillies Tendon Repair. Singapore Med J 2007 Aug;48(8):e234-6.

2. Nilsson-Helander K, Thurin A, Karlsson J, Eriksson BI. High incidence of venous thrombosis after Achillies tendon rupture: a prospective study. Knee Surg Sports Traumatol Arthrosc 2009;17(10):1234-8.

3. Patel A, Ogawa B, Charlton T, Thordarson D. Incidence of deep vein thrombosis and pulmonary embolism after Achilles tendon rupture. Clin Orthop Relat Res 2012 Jan;470(1):270-4.

4. Jørgensen PS, Warming T, Hansen $\mathrm{K}$, et al. Low molecular weight heparin (Innohep) as thromboprophylaxis in outpatients with a plaster cast: a venografic controlled study. Thromb Res 2002;105(6):477-80.
5. Lapidus LJ, Rosfors S, Ponzer S, et al. Prolonged thromboprophylaxis with dalteparin after surgical treatment of achilles tendon rupture: a randomized, placebo-controlled study. J Orthop Trauma 2007;21(1):52-7.

6. Lassen MR, Borris LC, Nakov RL. Use of Low molecular weight heparin reviparin to prevent deep -vein thrombosis after leg injury requiring immobilization. N Engl $\mathrm{J}$ Med 2002;347(10):726-30.

7. Magnusson M, Eriksson BI, Kalebo P, Sivertsson R. Is Colour Doppler Ultrasound A Sensitive Screening method in diagnosing deep vein thrombosis after hip surgery? Thromb Haemost 1996;75(2):242-5.

8. Schellong SM, Beyer J, Kakkar AK, et al. Ultrasound screening for asymptomatic deep vein thrombosis after major orthopaedic surgery: the VENUS study. J Thromb Haemost 2007;5(7):1431-7.

9. Kujath P, Spannagel U, Habscheid W. Incedence and prophylaxis of deep venous thrombosis in outpatiens with injury of the lower limb. Haemostasis 1993;23 Suppl 1:20-6.

10. NICE clinical guideline 92 for venousthromboembolism prophylaxis. Venous thromboembolism: reducing the risk January 2010:21.
Access full text article on other devices

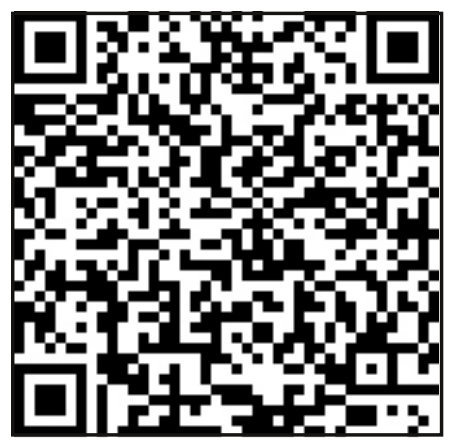

Access PDF of article on other devices

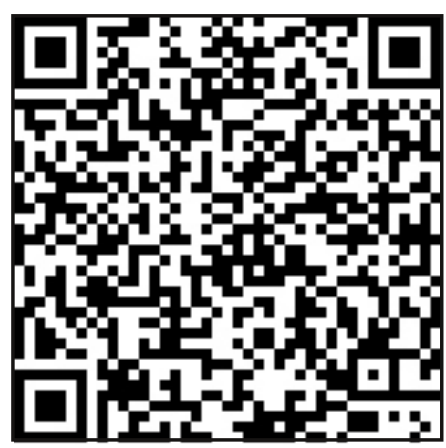

